\title{
Collective Intelligence Generation from user contributed content
}

Vassilios Solachidis ${ }^{1}$, Phivos Mylonas ${ }^{2}$, Andreas Geyer-Schulz ${ }^{3}$, Bettina Hoser $^{3}$, Sam Chapman ${ }^{4}$, Fabio Ciravegna ${ }^{4}$, Vita Lanfranchi ${ }^{4}$, Ansgar

Scherp $^{5}$, Stefen Staab ${ }^{5}$, Costis Contopoulos ${ }^{6}$, Ioanna Gkika ${ }^{6}$, Byron

Bakaimis $^{6}$, Pavel Smrz ${ }^{7}$, Yiannis Kompatsiaris ${ }^{1}$, and Yannis Avrithis ${ }^{2}$

1 Centre of Research and Technology Hellas, Informatics and Telematics Institute, Km Thermi-Panorama Road, Thermi-Thessaloniki, GR 570 01, Greece \{vsol, ikom\}@iti.gr

2 National Technical University of Athens, Image, Video and Multimedia Systems Laboratory, Iroon Polytechneiou 9, Zographou Campus, Athens, GR 157 80,

Greece \{fmylonas, iavr\}@image.ntua.gr

3 Department of Economics and Business Engineering, Information Service and Electronic Markets, Kaiserstraße 12, Karlsruhe 76128, Germany,

\{andreas.geyer-schulz, bettina.hoser\}@kit.edu

4 University of Sheffield, Department of Computer Science, Regent Court, 211 Portobello Street, S1 4DP, Sheffield, UK, \{s.chapman,

fabio,v.lanfranchi\}@dcs.shef.ac.uk

5 Universität Koblenz-Landau, Information Systems and Semantic Web, Universitätsstraße 1, 57070 Koblenz, Germany, \{staab, scherp\}@uni-koblenz.de

6 Vodafone-Panafon (Greece), Technology Strategic Planning - R\&D Dept., Tzavella 1-3, Halandri, 152 31, Greece \{Costis.Kontopoulos, Ioanna.Gkika, Byron.Bakaimis\}@vodafone.com

7 Brno University of Technology, Faculty of Information Technology, Bozetechova 2, CZ-61266 Brno, Czech Republic, smrz@fit.vutbr.cz

Summary. In this paper we provide a foundation for a new generation of services and tools. We define new ways of capturing, sharing and reusing information and intelligence provided by single users and communities, as well as organizations by enabling the extraction, generation, interpretation and management of Collective Intelligence from user generated digital multimedia content. Different layers of intelligence are generated, which together constitute the notion of Collective Intelligence. The automatic generation of Collective Intelligence constitutes a departure from traditional methods for information sharing, since information from both the multimedia content and social aspects will be merged, while at the same time the social dynamics will be taken into account. In the context of this work, we present two case studies an Emergency Response and a Consumers Social Group case study. 
Key words: Collective intelligence, media intelligence, social intelligence, mass intelligence, organizational intelligence, personal intelligence.

\section{Introduction}

The public has always played a major role in managing events in small and large communities, be they emergency events, the environment, or the organisation of public or private activities. The availability of mobile, networked information communication technologies in the hands of ordinary people makes information exchange increasingly potent and pervasive. The expected evolution for the near future is the evolution of Web based services supporting grassroots participation by users, customers and citizen in information sharing in a number of fields, from eCommerce (most of which are already ongoing, see [1], to emergency response [2] and consumer collective applications such as realtravel.com [3].

Existing Web technologies for multimedia annotation and sharing [4, 5], content creation [6], mass question answering [7,8] or social networking [9] provide exciting new opportunities to create innovative services. However, such approaches have reached a number of important limits in their evolution:

1. Inability to "understand" and, more specifically, the inability to manipulate the content automatically, leading to failure in making information available for further processing, and therefore failing to exploit the emergence of trends at the social and mass level, and the emergence of knowledge about a situation.

2. Limited access of such technology to mobile users and to organisations.

Also, the digital content rapidly reaches a mass that makes relevant information extremely complex and costly to handle. Yet, current applications do not fully support intelligent processing and management of such information. Thus, users fail to access it efficiently and cannot exploit the underlying knowledge.

In this paper novel techniques for exploiting multiple layers of intelligence from user-contributed content are presented. These layers, namely the Personal, Media, Mass, Social and Organizational Intelligence, constitute together the Collective Intelligence. They are a form of intelligence that emerges from the collaboration and competition among many individuals and that seemingly has a mind of its own. The decomposition of collective intelligence into five layers is a methodological approach for research and development that separates different concerns into orthogonal layers.

Collective Intelligence is extracted by understanding mass user-generated content with emphasis on integration and bridging (e.g. social and content dimensions) and the mobile and organizational - business aspects. This can be depicted by the following formula: 
Impact (Collective Intelligence) $>\sum$ Impact $\left(\right.$ Layer $\left._{i}\right), i \in I$

where $I$ is the set of layers of intelligence.

Also, collective intelligence benefits:

1. End users who will be able to receive personalised information based on Collective Intelligence in a largely automatic way.

2. Communities which will benefit from the generation of ad hoc services for their members and from improved community management

3. Organisations which will be able to manage different levels of intelligence to generate knowledge which will form the base of superior decisionsupport services.

4. Service providers, seeking new outlets for customer and market development, beyond provision of products focused on individuals.

To automate the acquisition of such knowledge from Collective Intelligence is a big departure from traditional methods for information sharing, since managing Collective Intelligence poses new requirements, for example, semantic analysis has to fuse information coming both from the content itself and the social context, and additionally, the social dynamics that emerge have to be taken into account.

In the context of this work, we shall present two case studies. Initially, an Emergency Response case study will be tackled, where users provide intelligence about large scale emergencies, empowering a more effective and informed emergency action and at the same time receive information on how to act. A Consumers Social Group case study will follow, providing enhanced publishing tools to support group activities (e.g. organization of team events) and the ability to extract meta-information from content sources and group discussions. Both Use Cases denote the important effect of Collective Intelligence as well as its leverage for private, commercial and public purposes.

In Section 2 the five layers that constitute the Collective intelligence will be described and. The Emergency Response and Consumers Social Group scenarios will be presented in Section 3 and the the conclusions in Section 4 .

\section{Collective Intelligence}

The five layers that constitute the Collective Intelligence will be described in the following subsections.

\subsection{Personal Intelligence}

The Personal Intelligence layer deals with enabling users to both upload and access multimedia information submitted to the intelligent services using a range of devices, from mobile phones to PDAs and personal computers. Once 
multimedia content is submitted to the intelligent services, a series of processing and analysis procedures take place in order to exploit, share and reuse the extracted knowledge. User and context modelling paradigms will be employed to enable personalised access to the content and knowledge available from the proposed applications.

Applications and services based on collective intelligence would be expected to have reached their peak of usage by 2015. Even though by that time many decisive factors such as the consumer trends on technology use (e.g. internet applications and live services), or the capabilities of future networks and terminals are expected to have changed, the user-centric interaction model that is expected to take place will remain the same.

This model will consist of a large number of "entities" like the actual events, the mechanisms of capturing event information (e.g. image and video cameras, geolocation possibilities, automatic recognition of voice annotations, etc.), the content types, the types of users, the devices, the network technologies, the network operators, the service providers, organisations, regulatory bodies etc. All these constitute the "degrees of freedom" that dictate the quality of services offered to the end users. In Figure 1 we present a rough representation of this model. It is effectively a user-centric interaction model or else, an end-to-end two-way information flow model.

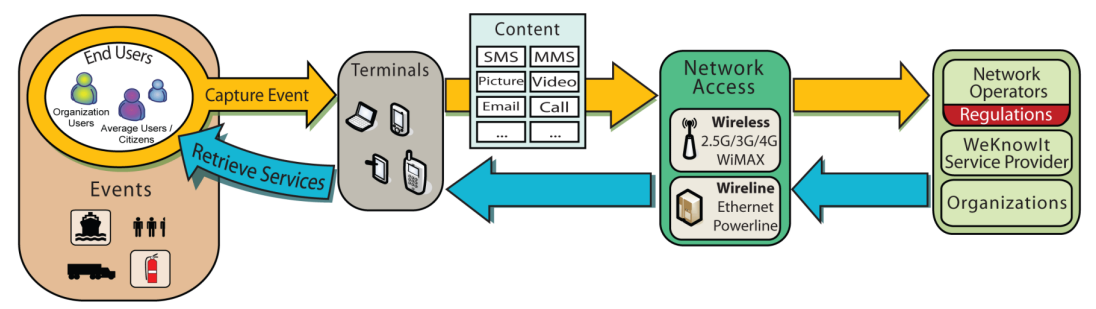

Fig. 1. High-level representation of the personal intelligence model

The rendition of an event by the end users, according to their perception and recording of it, is collected and sent to the system, so to be processed by the intelligent services. However, the uplink flow of this personal intelligence confronts limitations imposed by natural attributes of the elements through this flow. Specifically, due to the different types of users, the diverse mechanisms for content capture or the varying capabilities of devices and the access possibilities of the available networks, the actual information submitted to the system's algorithms may be demoted from what is expected or intended. This scaling down of information as perceived by the users being present at the time of an event, versus the quality of information that finally reaches the system is visually portrayed in Figure 2. This has implications in the quality of offered services, but to a great extent, is subject to the technological advances in the mobile devices and the wireless networks. 


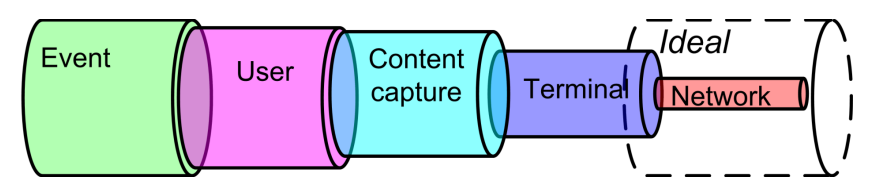

Fig. 2. Information Restriction Factors

\subsection{Media Intelligence}

The first and main step towards efficient "Media Intelligence" deals with automated analysis and semantics extraction from raw visual, textual or audio content and associated metadata. Analysis focuses on each modality in isolation and without taking into account any contextual information or the social environment. However, it does take into account prior knowledge, either implicit, in the form of supervised learning from training data, or explicit, in the form of knowledge driven approaches.

Extracting knowledge from raw data forms a huge research problem on its own so work in this field is expected to advance current existing state-ofthe-art techniques for each modality, while a significant effort will be devoted on

1. adapting to the individual domains of interest and intelligence methodologies,

2. handling heterogeneity of unstructured user-contributed resources and

3. supporting interoperability with contextual information.

Three main processes are proposed, text analysis, visual information analysis and speech analysis.

In text analysis process, textual information is of fundamental importance in every scenario where humans are involved. They are used to pass information explicitly to other people. Textual information is pervasive and - with the coming into existence of the Web - its availability is increasing. Intelligent techniques are required to enable automatic Information Extraction (IE) from text and make this information available for further processing.

Visual information, that is, still images and especially video, tend to impose huge requirements on current repositories or social networks in terms of storage or transmission due to the size of the data involved, yet its contribution to the knowledge and intelligence of related applications remains insignificant. Research in disciplines like image processing, pattern recognition and computer vision has been ongoing for decades but satisfactory performance can usually only be achieved in constrained domains, scales and environments.

Speech is a natural, pervasive and efficient means for communication among people. Therefore, it is the privileged modality in many situations where safety and convenience issues require hands- and eyes-free interaction with computers or ask for a direct access to information (no menu navigation, no typing). Its ubiquitous and easy-to-use character makes also speech the primary communication channel in emergency scenarios. 
In the context of media intelligence, we rather focus on realistic conditions that are compatible with the concept of collective intelligence. For example, when extracting information from a set of recorded phone calls in the emergency scenario, one can hardly expect the clean noise environment. The speech analysis will combine the standard large-vocabulary continuous speech transcription methods with advanced keyword-detection techniques based on phonetic search. In this way, the speech analysis part of the media intelligence services will be able to identify semantically relevant content (e.g., the names of people and places) that would not be accessible in traditional systems.

\subsection{Mass Intelligence}

Masses of users contribute their knowledge to communities in the Web 2.0. They organize and share media such as images on Flickr [4], videos on YouTube [5], bookmarks on Delicious [10], personal opinions, and others. Within such systems, the users can provide feedback by valuating the content provided and conduct assessments. This can be done, e.g., by participating in discussions and answering questions in a community portal.

Thus, Mass Intelligence combines the information from mass user feedback in order to extract patterns and trends that cannot be extracted by single content items. Facts and trends will be recognised and modelled by interpreting user feedback on a large scale. The key research challenge of Mass Intelligence is the questions whether this mass of users can give new insights that would not be possible by considering the individual. To this end, we are currently analyzing the Lycos $\mathrm{iQ}$ [8] data set. Users can ask arbitrary questions on the Lyocs iQ platform. The community answers questions in a discussion forum style. The answers are assessed by the community and credit points are awarded to the contributors. The Lycos iQ dataset analyzed for Mass Intelligence contains more than $900 \mathrm{k}$ questions in German language and $64 \mathrm{k}$ questions in English. For analyzing the dataset, four aspects are considered:

- Can mass question answering improve the quality of search results?

- Can implicit or explicit user feedback result in better ratings and rankings of questions?

- What semantics emerges from collaborative organization of media and knowledge by classification and clustering.

- How does the mass data categorization and mass behavior change over time and how do opinions evolve?

\subsection{Social Intelligence}

Social intelligence results from the monitoring, analysis, recognition, and understanding of the needs and capabilities of individuals and communities from their information usage and communication interaction patterns. Social intelligence delivers social information which may be used to improve other 
processes. At its simplest, social intelligence can be seen as a social markup process on actors and communication acts which provides social information as part of the pragmatic dimension of communication. For instance, consider the recognition of "hubs" in emergency situations such as during a hurricane. Providing these well-connected individuals with critical information will reach a broad set of people rapidly with minimal communication requirements, because the hubs are the individuals that spread messages most effectively. Or, consider the identification of authorities. In media intelligence or mass intelligence, content of these users should receive more emphasis and attention.

Watzlawicks's communication model [11] serves as the base for deriving the social intelligence layer. Social intelligence consists of three interconnected layers, namely the content layer of communication messages, the meta-layer of communication messages, and the structural information layer derived from social interaction which represents the state of the communication process in a community.

Social network analysis [12] and for directed social interactions a Hermitian eigensystem-analysis [13] serve as methods for analyzing the the interaction structure of social networks. For the semantic interpretation, social concepts from general sociology are used to interpret and assign meaning to the results of such an analysis. Modal extensions to the knowledge representation layer should be considered (see e.g. [14] and [15] For example, when doing an eigensystem-analysis of the link structure of the WWW, hubs and authorities are identified as concepts: hubs mean in that context web-sites that link to many relevant web-sites with the function to act as a multiplier, whereas authorities are the relevant web-sites with the important content. However, when analyzing the link structure in a social network site, the social concept changes, depending on the culture of the network, the relevant social concept may be that of friends, colleagues, or acquaintances. Furthermore, for the qualification of such interactions, the social position, role, rank of a person in his community is of high importance e.g. for marketing purposes. Visualized social structures may also serve as innovative interfaces to Internet communication services which ease the process of communicating with members of this social group.

Analysis at the meta-communication layer needs a strong link to mediaintelligence: For example, digital audio streams coming to a emergency callcenter may be analyzed for emotions. Recognizing emotions may help in evaluating the urgency of the situation. In an other setting, pictures about holiday resorts may be classified according to their emotional appeal. The exact wording of messages contains hints on the social background of the sender, so does the pronounciation of speech.

\subsection{Organizational Intelligence}

In contrast to Personal Intelligence, the Organizational Intelligence deals with the sharing of knowledge between the individual members of an organization. 
As a consequence, the role of Organizational Intelligence is to bring the right piece of knowledge at the right time to the right person of the organization in order to support decision making (cf. [16]). This knowledge is not necessarily produced by individuals, but rather by the interaction with Personal, Media, Mass, and Social Intelligence. The persons addressed with Organizational Intelligence can be either within the organization or member of an external organization. Traits of professional organizations such as enterprises and governmental agencies are strong and often legally enforceable rules and boundaries. The association of persons to the organization or parts of the organization are typically clearly defined such as a person being member of the $\mathrm{R} \& \mathrm{D}$ department, human resources, etc. In addition, also the role is typically known like head of group or silver command in emergency response. In contrast, non-professional organizations are only loosely coupled. The association of persons to the non-professional organization can be fuzzy such as being member of a neighborhood community or a group of friends. In addition, the roles may not be clearly defined in non-professional organizations. For example, for a group of friends that is planning and spending a weekend trip to a foreign city it is typically not clear or even defined who takes the organizer, leader, etc. role in the group.

The goal of Organizational Intelligence is to best support the professional as well non-professional organizations in carrying out its tasks and achieving its goals. To this end, we need to know the actors involved in the organization and how they are organized. We aim at modeling and managing the information flows and decision processes within the organizations. Here, we need to understand the decisions that are to be made, processes to be carried out, and which tasks are to accomplish to reach the goals.

\section{Use Cases}

The proposed system will demonstrate the wide applicability of its technologies through the design, implementation and evaluation of two heterogeneous case studies: an Emergency Response and a Consumers Social Group case study. These studies are complementary in terms of intended users, potential business model and social impact. Nevertheless, both of them will naturally build on top of the deployed technologies, and a common architecture.

\subsection{Emergency Response Case Study}

The Emergency Response case study aims to develop technologies and interaction modalities to better support professional users (i.e. Emergency Response personnel) and citizens involved in an emergency, by providing means to intelligently gather and reuse available knowledge, thus empowering a more effective and informed emergency action. With the increased use of mobile devices and digital cameras, people have become accustomed to capture events 
and share the information (e.g. the BBC news website for contributing to the news by uploading pictures or comments). We will design, implement and deliver technologies and methodologies enabling citizens distributed across the region to participate in the monitoring of an incident or event. This will benefit Emergency Response planners that will have real time information available on which they can base their decisions and strategies, enabling them to better react to an Emergency. Moreover, the system will automatically gather information available elsewhere on the network to aid the Emergency Response, thus making possible for an emergency planner to find exactly the needed knowledge amongst all the available information and to selectively make this knowledge available to the citizens (e.g. information about open roads, information about relatives involved, etc.) in a largely automated way. The technologies in use will therefore also encourage and enable dialogue between the Emergency Responders and individuals, groups and communities The case study will be based on the research results of all Intelligence Layers, with Media, Mass and Social Intelligence analysing the user-submitted content, Personal Intelligence allowing easier upload and distribution of content to the end users and Organisational Intelligence providing all the extracted knowledge to the Emergency Responders.

\subsection{Consumers Social Group Case Study}

A case study of the consumer social group application will be the implementation of a travel planner and guide. This application is intended as a travel adviser during the whole lifecycle of a trip: from planning until the end of it. This web-based service will draw travel-related information from multiple sources, such as: history of previous trips (including information extracted from trip reports, like photographs, videos, or text and voice annotations), user feedbacks from blogs about visits of other people, or information available in the internet. Analysis of this information at different levels of intelligence leads to collective intelligence that can assist the service users in deciding and scheduling their future trip. This will result based on user-posed criteria, such as destination preferences, time-of-the-year suggestions, social group members with whom to travel, logistics and cost of travel and stay, special enquiries about events, attractions, museums, restaurants, shopping, nightlife, etc. Once the group agrees on their trip plan, the users can carry the details of it during their trip, in their portable devices (e.g. PDA, smart phones). At the time of their trip, the users can ask the system for recommendations about specific locations, events, etc., also with reference to the vicinity of their current location. Besides, they can use their mobile devices in order to capture instances of their trip (through photos, video footages, annotations, etc.). This content can either be uploaded to the system's server, at once, or be locally stored in the device, so as to be synchronized with the server at a later time. Once the trip comes to an end, the users can post their report about their trip experience, at the ease of their desk, for instance. This information will feed 
the system with new material to be processed and analysed, for future trip planning.

\section{Conclusions}

In this paper novel techniques for exploiting multiple layers of intelligence from user-contributed content have been presented. These layers constitute together the Collective Intelligence. Collective Intelligence provides added value to the available information, enabling the accomplishment of tasks that are not possible otherwise and time reduction and enhanced efficiency in existing procedures and workflows.

\section{Acknowledgement}

The research leading to these results has received funding from the European Community's Seventh Framework Programme FP7/2007-2013 under grant agreement $n^{\circ} 215453$ - WeKnowIt.

\section{References}

1. T. OReally, What is Web 2.0: Design Patterns and Business Models for the Next Generation of Software (OReilly Media Inc, September 2005).

2. L. Palen, S. Hiltz and S. Liu, Communications of the ACM 50 (2007) 54.

3. R. Conrady, Travel technology in the era of Web 2.0Trends and Issues in Global Tourism 2007 (Springer Berlin Heidelberg, 2007).

4. Flickr, http://www.flickr.com.

5. Youtube, http://www.youtube.com.

6. Wikipedia, http://www.wikipedia.com.

7. Yahoo, http://answers.yahoo.com.

8. Lycos, http://iq.lycos.co.uk.

9. Facebook, http://www.facebook.com.

10. Delicious, http://delicious.com/.

11. P. Watzlawick, J.H. Beavin and D.D. Jackson, Menschliche Kommunikation: Formen, St "orungen, Paradoxien" (Hans Huber, Bern, 1974).

12. S. Wasserman and K. Faust, Social Network Analysis, Structural Analysis in the Social Sciences Vol. 8, 1 ed. (Cambridge University Press, Cambridge, 1999).

13. A.G.S. B. Hoser, Journal of Mathematical Sociology 29 (2005) 265 .

14. P. Blackburn, Modal LogicCambridge Tracts in Theoretical Computer Science (Cambridge University Press, Cambridge, 2002).

15. M. Fitting and R. Mendelsohn, First-Order Modal Logic, Synthese Library Vol. 277 (Kluwer Academic, Dordrecht, 1999).

16. G.T. Schreiber and H. Akkermans, Knowledge engineering and management: the CommonKADS methodology (MIT Press, Cambridge, MA, USA, 2000). 